\title{
Age- and Gender-Specific Prevalence of Risk Factors in Patients with First-Ever Ischemic Stroke in China
}

\author{
Xiao-ying Yao, Yan Lin, Jie-li Geng, Ya-meng Sun, \\ Ying Chen, Guo-wen Shi, Qun Xu, and Yan-sheng Li \\ Department of Neurology, Renji Hospital, Shanghai Jiaotong University School of Medicine, Shanghai 200127, China \\ Correspondence should be addressed to Yan-sheng Li, lliyans@hotmail.com
}

Received 10 January 2012; Revised 18 March 2012; Accepted 29 April 2012

Academic Editor: Janika Kõrv

Copyright (c) 2012 Xiao-ying Yao et al. This is an open access article distributed under the Creative Commons Attribution License, which permits unrestricted use, distribution, and reproduction in any medium, provided the original work is properly cited.

\begin{abstract}
Background. Evidences are accumulating that age and gender have great impact on the distribution of stroke risk factors. Such data are lacking in Chinese population. Methods. 1027 patients with first-ever ischemic stroke (IS) were recruited and divided into young adult ( $<50$ years), middle-aged ( $50 \sim 80$ years), and very old ( $>80$ years) groups according to stroke onset ages. Vascular risk factors were collected and compared among groups. Results. Female patients were globally older than male patients at stroke onset and having higher prevalence of diabetes mellitus (DM), heart diseases, and atrial fibrillation (AF). However, females were less likely to drink heavily or smoke than males. Young patients had a much higher proportion of smoking and drinking than middleaged and very old patients and the highest family history of hypertension, while very old patients had the highest prevalence of heart diseases and AF but lowest proportion of positive family history of vascular diseases. Hypertension and DM were equally frequent among three groups. Conclusion. Our study showed that vascular risk factors had a specific age and gender distribution pattern in Chinese IS patients. Secondary prevention strategy should emphasize on the control of different risk factors based on patient's age and gender.
\end{abstract}

\section{Introduction}

Cerebrovascular disease ranks as the first leading cause of death in China according to the recent report of the global relative burden of stroke [1] and the major cause of disability in adolescent [2], with up to half of all patients who survive a stroke failing to regain independence and needing longterm health care [3]. Stroke can affect individuals of any age, although the incidence and prevalence of this disease increase sharply with age [4]. Prior data from both western countries and China disclose a higher incidence of ischemic stroke in men than in women in patients under 80 , however, most very old patients with stroke (aged $>80$ years) are women $[5,6]$. In addition, disparities exist in risk factors, clinical presentation, and outcomes of stroke among patients with different ages and genders [6-11].

Most ischemic stroke patients have multiple vascular risk factors and it is ultimately important in the primary and secondary preventive strategies to recognize and control them. There are quite a few studies from developed countries concerning the age and gender effect on the profile of vascular risk factors in ischemic stroke patients and the results are inconsistent $[5,12-14]$. Studies of a cohort of over 2600 ischemic stroke patients from a single Chinese center also revealed a relatively distinguished pattern of risk factors between male and female patients and between patients over 80 and under $80[6,11]$, but such studies are scarce in Chinese population and whether the distribution pattern is different from that of developed countries remains to be elucidated. Thereby, we investigated the age- and genderspecific prevalence of vascular risk factors in patients with first-ever ischemic stroke in Shanghai, a metropolitan urban area in China.

\section{Methods}

From January 2004 to December 2009, patients admitted to the Department of Neurology of Shanghai Renji Hospital, with first-ever acute ischemic stroke confirmed by cranial 
CT or MRI within 14 days after onset were, retrospectively, reviewed. Renji Hospital is a teaching hospital affiliated to Shanghai Jiaotong University School of Medicine and its stroke center is one of the largest centers in Shanghai with around 800 to 1000 patients visiting stroke clinic and around 100-150 stroke patients sending to emergency room every week.

Data on the following risk factors were collected: (1) smoking history (including both current and exsmoking); (2) heavy alcohol consumption ( $\geq 2$ standard alcohol consumption per day); (3) hypertension (including documented history of hypertension or undertaking antihypertensive drugs); (4) diabetes mellitus (documented medical record of diabetes mellitus or taking oral antidiabetic agents/insulin before the stroke); (5) heart diseases (including coronary heart diseases, arrhythmia and chronic heart failure); (6) atrial fibrillation; (7) family history of cerebrovascular and/or cardiovascular diseases. Systematic investigations were performed during the admission time, including brain CT and/or MRI, carotid ultrasound, 12 lead ECG, as well as standard blood and urine tests that including fasting serum glucose, cholesterol, and triglyceride.

All patients were divided into three age groups according their ages at onset: (1) young adult group ( $<50$ years); (2) middle-aged group (50 80 years); (3) very old group ( $>80$ years).

Categorical variables were summarized as counts (percentage) and continuous variables as means (standard deviation (SD)) or medians (interquartile ranges (IQR)), if not distributed normally. We performed univariate analyses of continuous variables using Manne-Whitney test and categorical variables using the chi-square test. The Cochran-MantelHaenszel test was used to carry out adjustment for age groups when testing the association between gender and risk factors. A two-tailed probability value $<0.05$ was considered significant. Data analysis was performed using SPSS version 18.0 (SPSS Inc., Chicago, III, USA).

\section{Results}

3.1. General Features. One thousand and twenty-seven patients with first-ever ischemic stroke were included in our study. Their demographic characteristics and vascular risk factors were summarized in Table 1 . Overall, $60.5 \%$ of these patients were male. The median age (IQR) was 67.5 (57.674.7) years.

3.2. The Comparison of Risk Factor Profiles between Male and Female Patients. Female patients were much older than male patients $(71.1$ versus 65.0 years, $P<0.001$ ) when experiencing their first ischemic stroke attack and had a significantly higher prevalence of diabetes mellitus $(P=$ $0.004)$, heart diseases $(P<0.001)$, and atrial fibrillation $(P=0.009)$. In contrast, male patients were more likely to be current or exsmokers $(P<0.001)$ and to drink heavily $(P<0.001)$ than female patients. Hypertension was equally frequent in both groups. With regard to family history, more male patients had positive family history of hypertension than female patients $(P=0.040)$ while the family history of heart diseases and stroke showed no significant differences (Table 1).

3.3. The Comparison of Risk Factor Profiles in Three Age Groups. The median (IQR) ages of the young adult group, the middle-aged group, and the very old group were 47.3 (43.7-48.8), 67.0 (59.5-73.2), and 83.0 (81.5-85.6) years, respectively, range 25.1-49.9, 50.1-79.9, and 80.0-102.5, years, respectively. The results of the comparison among the three age groups were displayed in Table 2 .

The incidence of ischemic stroke in patients $<80$ years of age was higher in men than in women. Most patients in the very old group, however, are women. Young adult patients had a much higher proportion of smoking and drinking than the other two groups, while very old patients had a much lower proportion of smoking and drinking than the other two group patients. So an increasing prevalence of smoking and heavy drinking with the lowering of age at stroke onset was observed.

Hypertension and diabetes were equally prevalent in three age groups, which suggested these two risk factors were common and consistent in ischemic stroke patients with all age ranges, while heart diseases were more frequent in very old patients.

Family history of hypertension was more frequent in young adults than in middle-aged and very old patients, whereas very old patients were less likely to have positive family history of hypertension, heart diseases, or stroke. It suggested a genetic component in the pathogenesis of ischemic stroke in young patients. In other words, patients with positive family history of hypertension, heart diseases, or stroke tended to have stroke attack at an earlier age.

3.4. The Comparison of Risk Factor Profiles between Male and Female Patients in Different Age Groups. In the young adult group, discrepancies of the distribution of risk factors were presented between male and female patients (Table 3). Male patients were predominantly more likely to be current or past smokers and heavy drinkers than female patients, whereas more females had heart diseases $(P=0.015)$. No difference could be observed in the family history of hypertension, heart diseases, or stroke between males and females.

In the middle-aged group, smoking and drinking history were still more prevalent in male than in female patients, but female patients were more likely to have diabetes, heart diseases, and atrial fibrillation $(P=0.004,0.001$, and 0.039 , resp.).

In the very old group, we could not find any difference between two genders in the distributed pattern of vascular risk factors except that males had a higher frequency of smoking and drinking.

\section{Discussion}

A few large-scale studies carried out in western countries revealed that there were complex interactions among age, 
TABLE 1: The comparison of demographic characteristics and vascular risk factors between female and male patients.

\begin{tabular}{lccc}
\hline & Total $(n=1027)$ & Male $(n=621)$ & Female $(n=406)$ \\
\hline Age (years) & $67.5(57.6-74.7)$ & $65.0(11.5)$ & $71.1(60.7-76.8)^{*}$ \\
Smoking & $38.0 \%$ & $59.9 \%$ & $4.4 \%^{*}$ \\
Heavy alcohol consumption & $19.5 \%$ & $31.6 \%$ & $1.0 \%^{*}$ \\
Hypertension & $66.2 \%$ & $64.3 \%$ & $69.2 \%$ \\
Diabetes mellitus & $22.2 \%$ & $19.2 \%$ & $26.8 \%^{*}$ \\
Heart diseases & $23.0 \%$ & $19.2 \%$ & $28.8 \%^{*}$ \\
Atrial fibrillation & $5.4 \%$ & $3.9 \%$ & $7.6 \%^{*}$ \\
Family history & & & $41.6 \% *$ \\
$\quad$ Hypertension & $45.6 \%$ & $48.1 \%$ & $13.8 \%$ \\
$\quad$ Heart diseases & $13.1 \%$ & $12.7 \%$ & $23.4 \%$ \\
$\quad$ Stroke & $24.8 \%$ & $25.8 \%$ & \\
\hline
\end{tabular}

${ }^{*} P<0.05$.

TABLE 2: The comparison of vascular risk factors in three age groups.

\begin{tabular}{lcccc}
\hline & Total $(n=1027)$ & $\begin{array}{c}\text { Young adult group } \\
(n=98)\end{array}$ & $\begin{array}{c}\text { Middle-aged group } \\
(n=801)\end{array}$ & $\begin{array}{c}\text { Very old group } \\
(n=128)\end{array}$ \\
\hline Male & $60.5 \%$ & $69.4 \%$ & $61.5 \%$ & $46.9 \%^{\# \dagger}$ \\
Smoking & $38.0 \%$ & $55.1 \%^{*}$ & $38.5 \%$ & $21.9 \%^{\# \dagger}$ \\
Heavy alcohol consumption & $19.5 \%$ & $32.7 \%^{*}$ & $20.0 \%$ & $6.3 \%^{\# \dagger}$ \\
Hypertension & $66.2 \%$ & $70.4 \%$ & $65.5 \%$ & $67.2 \%$ \\
Diabetes mellitus & $22.2 \%$ & $15.3 \%$ & $23.1 \%$ & $21.9 \%$ \\
Heart diseases & $23.0 \%$ & $7.1 \% \%^{*}$ & $23.2 \%$ & $33.6 \%^{\# \dagger}$ \\
Atrial fibrillation & $5.4 \%$ & $1.0 \%$ & $5.2 \%$ & $9.4 \%^{\dagger}$ \\
Family history & & & & \\
$\quad$ Hypertension & $45.6 \%$ & $63.3 \% *$ & $46.9 \%$ & $23.4 \%^{\# \dagger}$ \\
$\quad$ Heart diseases & $13.1 \%$ & $18.4 \%$ & $14.1 \%$ & $3.1 \%^{\# \dagger}$ \\
$\quad$ Stroke & $24.8 \%$ & $33.7 \%$ & $25.6 \%$ & $13.3 \%^{\# \dagger}$ \\
\hline
\end{tabular}

${ }^{*} P<0.05$ versus middle-aged group; ${ }^{\#} P<0.05$ versus middle-aged group; ${ }^{\dagger} P<0.05$ versus young adult group.

gender, and prevalence of stroke risk factors $[8,9,12]$. These results are not completely consistent and differ between countries and ethnics. Thus, it becomes increasingly important to find out the age- and gender-specific stroke risk factors in Chinese population in order to develop better stroke prevention strategies.

In this group of 1027 Chinese first-ever ischemic stroke patients, women were globally older than men at the stroke onset; had less unhealthy lifestyles such as smoking and heavy drinking, however, were more likely to have diabetes, heart diseases, and atrial fibrillation than men. Hypertension, the most important risk factor for ischemic stroke, was equally common in both genders. As for the age effect, younger patients were more frequent to have an unhealthy lifestyles and positive family history of cardiovascular or cerebrovascular diseases, while older patients had higher prevalence of classic vascular risk factors such as ischemic heart diseases, chronic heart failure, and atrial fibrillation.

Currently, the wide accepted risk factors for ischemic stroke include old age, male sex, hypertension, diabetes mellitus, dyslipidemia, atrial fibrillation, smoking, heavy drinking, et al. The INTERSTROKE study, a standardized case-control study carried out in 22 countries worldwide, showed that 12 risk factors including hypertension $(\mathrm{OR}=2.37)$, current smoking $(\mathrm{OR}=2.32)$, waist-to-hip ratio $(\mathrm{OR}=1.65)$, unhealthy diet $(\mathrm{OR}=1.29)$, regular physical activity $(\mathrm{OR}=0.68)$, diabetes mellitus $(\mathrm{OR}=1.60)$, alcohol intake $(\mathrm{OR}=1.41)$, psychosocial stress $(\mathrm{OR}=1.30)$, depression $(\mathrm{OR}=1.47)$, cardiac causes $(\mathrm{OR}=2.74)$, and ratio of apolipoproteins B to A1 $(\mathrm{OR}=1.30)$ collectively accounted for $89.7 \%$ of the population-attributable risks for ischemic stroke [15]. The present study found that men were generally younger than women at their first attack of ischemic stroke and there is a higher percentage of male among all ischemic stroke patients. The results were consistent with prior data from consecutive inception cohorts from China [10, 11], which also revealed a relatively younger onset age in male ischemic stroke patients and a nearly $60: 40$ split of male and female in all recruited patients. However, our data and previous studies $[5,11]$ all showed that women predominate in the very old group of ischemic stroke patients. With the proportion of elderly individuals increasing rapidly, ischemic 
TABLE 3: The comparison of vascular risk factors between male and female patients in each age group.

\begin{tabular}{|c|c|c|c|c|c|c|}
\hline & \multicolumn{2}{|c|}{$<50$ years } & \multicolumn{2}{|c|}{$50-80$ years } & \multicolumn{2}{|c|}{$>80$ years } \\
\hline & Male $(n=68)$ & Female $(n=30)$ & Male $(n=493)$ & Female $(n=308)$ & Male $(n=60)$ & Female $(n=68)$ \\
\hline Smoking history & $77.9 \%$ & $3.3 \%{ }^{*}$ & $60.0 \%$ & $3.9 \% *$ & $38.3 \%$ & $7.4 \% *$ \\
\hline Heavy alcohol consumption & $44.1 \%$ & $6.7 \% *$ & $32.0 \%$ & $0.6 \% *$ & $13.3 \%$ & $0 \% *$ \\
\hline Hypertension & $67.6 \%$ & $76.7 \%$ & $62.5 \%$ & $70.5 \%$ & $75.0 \%$ & $60.3 \%$ \\
\hline Diabetes mellitus & $14.7 \%$ & $16.7 \%$ & $19.7 \%$ & $28.6 \% *$ & $20.0 \%$ & $23.5 \%$ \\
\hline Heart diseases & $2.9 \%$ & $16.7 \% *$ & $19.5 \%$ & $29.2 \% *$ & $35.0 \%$ & $32.4 \%$ \\
\hline Atrial fibrillation & $1.5 \%$ & $0 \%$ & $3.9 \%$ & $7.5 \% *$ & $6.7 \%$ & $11.8 \%$ \\
\hline \multicolumn{7}{|l|}{ Family history } \\
\hline Hypertension & $60.3 \%$ & $70.0 \%$ & $49.3 \%$ & $43.2 \%$ & $25.0 \%$ & $22.1 \%$ \\
\hline Heart diseases & $13.2 \%$ & $30.0 \%$ & $13.8 \%$ & $14.6 \%$ & $3.3 \%$ & $2.9 \%$ \\
\hline Stroke & $32.4 \%$ & $36.7 \%$ & $26.2 \%$ & $24.7 \%$ & $15.0 \%$ & $11.8 \%$ \\
\hline
\end{tabular}

${ }^{*} P<0.05$.

stroke will represent a massive epidemic in the forthcoming years, especially in women. So this female subgroup is becoming a specific healthcare issue for society.

Previous studies had consistently reported that there was a significantly higher proportion of smokers and heavy drinkers in male than in female patients $[16,17]$. We found such a phenomenon in all age groups in the present cohort, furthermore, the younger the age, the higher the proportion of smokers and drinkers in male patients. These findings suggest that the change of unhealthy lifestyles is of great importance to prevent ischemic stroke in men, especially in young men, which also brings public health education an urgent issue.

In the present study, hypertension is the most common risk factor for ischemic stroke. Its prevalence showed no significant difference between two gender groups or in all age groups. A meta-analysis including 45 studies and representing a total of 673,935 ischemic stroke patients showed that women had more hypertension than men $(\mathrm{OR}=$ 1.17, 95\% CI: $1.03-1.34, P=0.017$ ) [18]. However, there are also studies showed that hypertension became increasingly (although slightly) common in women after the age of 65 whereas the prevalence of it decreased in men after that age $[12,19]$. The discord between different studies may be resulted from the different racial and environmental factors in the pathogenesis of hypertension.

We noted that female patients were more frequently to have diabetes mellitus, mainly in patients aged 50 to 80 , which is similar to the results of other studies carried out in Chinese and African American [10, 11, 20], but obviously opposite to most studies in European and White American $[13,16,17]$. The real reason for this discrepancy is unknown and probably owing to genetic liability and different life styles.

In agreement with other study results [13, 16, 17], we observed a higher incidence of atrial fibrillation in female patients than in male; moreover, atrial fibrillation was far more common in patients over 80 than under the age of 50 . As reported by Framingham Heart Study and other related studies, women are more susceptible to atrial fibrillation $[18,21]$, thus taking higher risk for cardiogenic embolism [22]. To be more specified, Roquer et al. figured out through their work that the risk of cardiogenic embolism in women was twice as much as that in men [14]. The progressive loss of conduction tissue cells and pacemaker function accounts for the increased incidence of atrial fibrillation in the elderly [23]. There is a high prevalence of atrial fibrillation and heart diseases (including coronary heart diseases, arrythmia and chronic heart failure) in elderly patients, which makes cardiogenic embolism a more frequent cause of ischemic stroke [24]. Some studies suggested that atrial fibrillation might supercede hypertension to be the primary risk factor for ischemic stroke in patients aged over 80 or 85 years [25]. Therefore, early recognition and intensive control of heart diseases, especially using warfarin, are of critical importance in reducing the incidence of ischemic stroke in the elderly.

With regards to the analysis of the family history in firstever ischemic stroke patients, we found that young patients aged under 50 were more likely to have positive family history of hypertension, heart diseases, or stroke. Family history was reported as the second most common risk factor (18.4\%) by De Silva et al. in young stroke patients in Sri Lanka [26]. Also, Rasura et al. found a positive family history of vascular events in $63 \%$ of young stroke patients [27]. A significant genetic component underlying the occurrence of young stroke was demonstrated in prior studies and metaanalysis of all published twin studies on stroke [28, 29]. These findings have important value in stroke prevention because it is presumed that those who have positive family history of vascular events will impressively perceive the harm of stroke early in their lives and realize the importance of early prophylaxis. Thus, they might be easier to be educated to change their unhealthy lifestyles and have a good compliance. In another way, they are supposed to be the main target population for primary stroke prevention.

The present study has inevitable limitations. Firstly, it was carried out in a single stroke center (although one of the largest) in Shanghai and thus could not stand for the whole population of ischemic stroke patients. Secondly, patients with minor symptoms or fatal events (especially in very old patients) might not be able to be admitted to our hospital, which could cause selection bias. Finally, the analysis of risk factor profiles was incomplete in this study for not collecting other possible stroke risk factors such as the body mass index, serum homocysteine, peripheral vascular diseases, psychogenic factors, and severity of atherosclerosis (such as the 
intima-media thickness of carotid arteries and the stenosis of intra- and extracranial arteries).

Despite these limitations, however, the present study still provided some valuable information on the distribution of risk factors in first-ever ischemic stroke patients with different genders and ages. The identification of risk factor profiles in different groups of ischemic stroke patients is essential before the draft of etiologic preventive strategies. According to the findings in present study, the priority in stroke prevention for men and young (especially young men) should be the change of lifestyles such as giving up smoking and alcohol drinking. As for women and the elderly, it is much more important to treat traditional vascular risk factors such as hypertension and diabetes mellitus. The recognition and control of atrial fibrillation should also be taken seriously in elderly population. Additionally, young adults with a positive family history of vascular events should be educated early to control risk factors effectively.

\section{Conclusions}

Overall, the present study was one of the very few studies to demonstrate the age and gender specific distribution of risk factors in ischemic stroke patients in Chinese population and found out that female and male, young and old patients had very different stroke risk factor profiles. Population based epidemiological studies are warrented to a better understanding of this distribution pattern.

\section{References}

[1] A. S. Kim and S. C. Johnston, "Global variation in the relative burden of stroke and ischemic heart disease," Circulation, vol. 124, no. 3, pp. 314-323, 2011.

[2] C. J. L. Murray and A. D. Lopez, "Global mortality, disability, and the contribution of risk factors: global burden of disease study," The Lancet, vol. 349, no. 9063, pp. 1436-1442, 1997.

[3] J. W. Sturm, G. A. Donnan, H. M. Dewey et al., "Quality of life after stroke: the North East Melbourne stroke incidence study (NEMESIS)," Stroke, vol. 35, no. 10, pp. 2340-2345, 2004.

[4] C. Marini, L. Triggiani, N. Cimini et al., "Proportion of older people in the community as a predictor of increasing stroke incidence," Neuroepidemiology, vol. 20, no. 2, pp. 91-95, 2001.

[5] J. I. Rojas, M. C. Zurrú, M. Romano, L. Patrucco, and E. Cristiano, "Acute ischemic stroke and transient ischemic attack in the very old-risk factor profile and stroke subtype between patients older than 80 years and patients aged less than 80 years," European Journal of Neurology, vol. 14, no. 8, pp. 895899, 2007.

[6] D. Wang, Z. Hao, W. Tao et al., "Acute ischemic stroke in the very elderly Chinese: risk factors, hospital management and one-year outcome," Clinical Neurology and Neurosurgery, vol. 113, no. 6, pp. 442-446, 2011.

[7] S. Olindo, P. Cabre, R. Deschamps et al., "Acute stroke in the very elderly: epidemiological features, stroke subtypes, management, and outcome in Martinique, French West Indies," Stroke, vol. 34, no. 7, pp. 1593-1597, 2003.

[8] M. Niewada, A. Kobayashi, P. A. Sandercock, B. Kamiński, and A. Członkowska, "International stroke trial collaborative group. Influence of gender on baseline features and clinical outcomes among 17,370 patients with confirmed ischemic stroke in the international stroke trial," Neuroepidemiology, vol. 24, no. 3, pp. 123-128, 2005.

[9] C. Ayala, J. B. Croft, K. J. Greenlund et al., "Sex differences in US mortality rates for stroke and stroke subtypes by race/ethnicity and age, 1995-1998," Stroke, vol. 33, no. 5, pp. 1197-1201, 2002.

[10] Y. Huang, J. G. Wang, J. W. Wei et al., "Age and gender variations in the management of ischaemic stroke in China," International Journal of Stroke, vol. 5, no. 5, pp. 351-359, 2010.

[11] F. Y. Kong, W. D. Tao, Z. L. Hao, and M. Liu, "Predictors of one-year disability and death in Chinese hospitalized women after ischemic stroke," Cerebrovascular Diseases, vol. 29, no. 3, pp. 255-262, 2010.

[12] K. K. Andersen, Z. J. Andersen, and T. S. Olsen, "Age- and gender-specific prevalence of cardiovascular risk factors in 40 102 patients with first-ever ischemic stroke: a Nationwide Danish Study," Stroke, vol. 41, no. 12, pp. 2768-2774, 2010.

[13] D. Giralt, S. Domingues-Montanari, M. Mendioroz et al., "The gender gap in stroke: a meta-analysis," Acta Neurologica Scandinavica, vol. 125, no. 2, pp. 83-90, 2012.

[14] J. Roquer, A. Rodríguez Campello, and M. Gomis, "Sex differences in first-ever acute stroke," Stroke, vol. 34, no. 7, pp. 1581-1585, 2003.

[15] M. J. O’Donnell, X. Denis, L. Liu et al., "Risk factors for ischaemic and intracerebral haemorrhagic stroke in 22 countries (the INTERSTROKE study): a case-control study," The Lancet, vol. 376, no. 9735, pp. 112-123, 2010.

[16] M. J. Reeves, C. D. Bushnell, G. Howard et al., "Sex differences in stroke: epidemiology, clinical presentation, medical care, and outcomes," The Lancet Neurology, vol. 7, no. 10, pp. 915926, 2008.

[17] M. J. Reeves, G. C. Fonarow, X. Zhao, E. E. Smith, and L. H. Schwamm, "Quality of care in women with ischemic stroke in the GWTG program," Stroke, vol. 40, no. 4, pp. 1127-1133, 2009.

[18] J. P. Broderick, S. J. Phillips, W. M. O'Fallon, R. L. Frye, and J. P. Whisnant, "Relationship of cardiac disease to stroke occurrence, recurrence, and mortality," Stroke, vol. 23, no. 9, pp. 1250-1256, 1992.

[19] National Centre for Health Statistics, Health, United States, 2009: With Special Feature on Medical Technology, National Centre for Health Statistics, Hyattsville, Md, USA, 2010.

[20] M. Eriksson, E. L. Glader, B. Norrving, A. Terént, and B. Stegmayr, "Sex differences in stroke care and outcome in the swedish national quality register for stroke care," Stroke, vol. 40, no. 3, pp. 909-914, 2009.

[21] J. M. Holroyd-Leduc, M. K. Kapral, P. C. Austin, and J. V. $\mathrm{Tu}$, "Sex differences and similarities in the management and outcome of stroke patients," Stroke, vol. 31, no. 8, pp. 18331837, 2000.

[22] H. S. Cabin, K. S. Clubb, C. Hall, R. A. Perlmutter, and A. R. Feinstein, "Risk for systemic embolization of atrial fibrillation without mitral stenosis," American Journal of Cardiology, vol. 65, no. 16, pp. 1112-1116, 1990.

[23] A. Shuaib and V. C. Hachinski, "Mechanisms and management of stroke in the elderly," Canadian Medical Association Journal, vol. 145, no. 5, pp. 433-443, 1991.

[24] A. Arboix, M. Miguel, E. Císcar, L. García-Eroles, J. Massons, and M. Balcells, "Cardiovascular risk factors in patients aged 85 or older with ischemic stroke," Clinical Neurology and Neurosurgery, vol. 108, no. 7, pp. 638-643, 2006.

[25] E. Auriel, A. Y. Gur, O. Uralev et al., "Characteristics of first ever ischemic stroke in the very elderly: profile of vascular risk 
factors and clinical outcome," Clinical Neurology and Neurosurgery, vol. 113, no. 8, pp. 654-657, 2011.

[26] R. De Silva, R. Gamage, C. Wewelwala et al., "Young strokes in Sri Lanka: an unsolved problem," Journal of Stroke and Cerebrovascular Diseases, vol. 18, no. 4, pp. 304-308, 2009.

[27] M. Rasura, A. Spalloni, M. Ferrari et al., "A case series of young stroke in Rome," European Journal of Neurology, vol. 13, no. 2, pp. 146-152, 2006.

[28] E. Floßmann, U. G. R. Schulz, and P. M. Rothwell, "Systematic review of methods and results of studies of the genetic epidemiology of ischemic stroke," Stroke, vol. 35, no. 1, pp. 212-227, 2004.

[29] L. M. Brass, J. L. Isaacsohn, K. R. Merikangas, and C. D. Robinette, "A study of twins and stroke," Stroke, vol. 23, no. 2, pp. 221-223, 1992. 


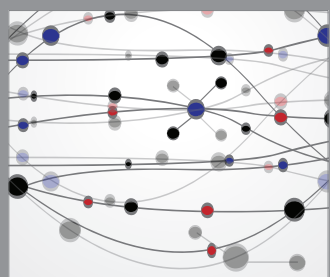

The Scientific World Journal
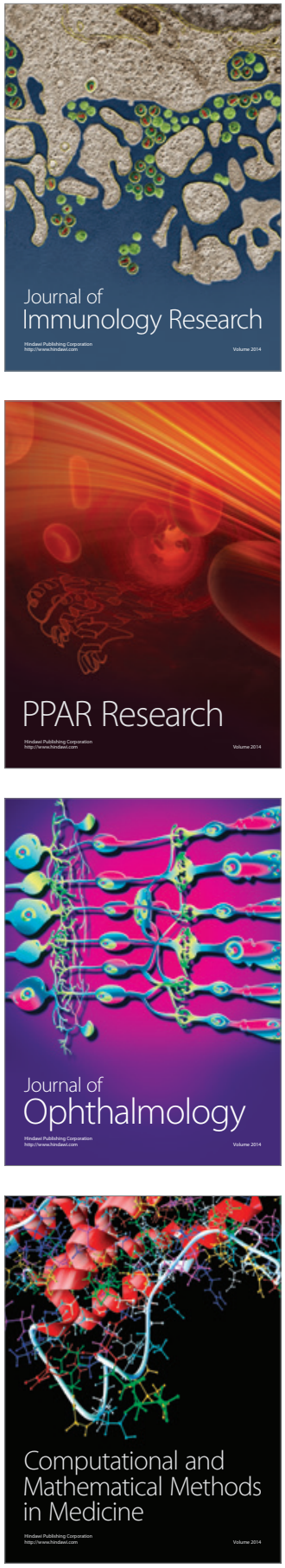

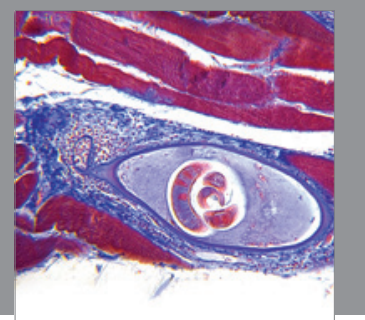

Gastroenterology

Research and Practice
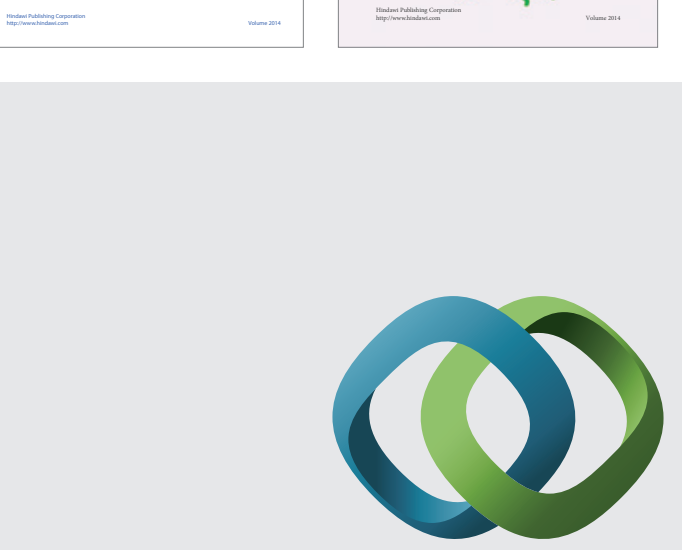

\section{Hindawi}

Submit your manuscripts at

http://www.hindawi.com
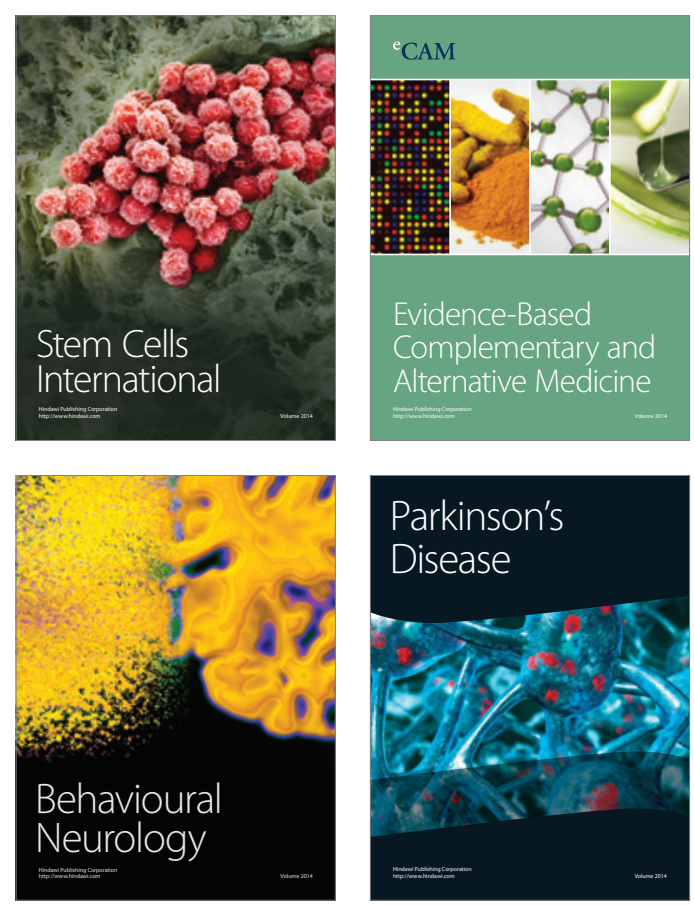

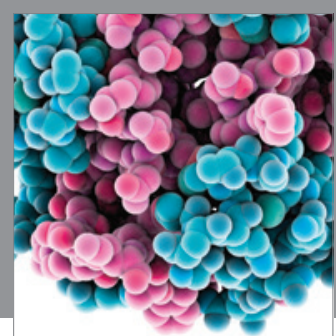

Journal of
Diabetes Research

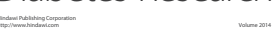

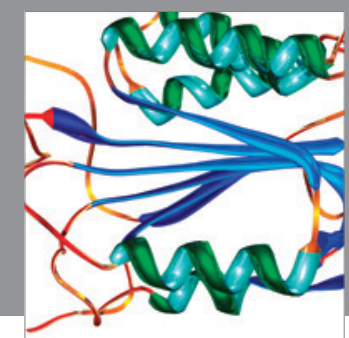

Disease Markers
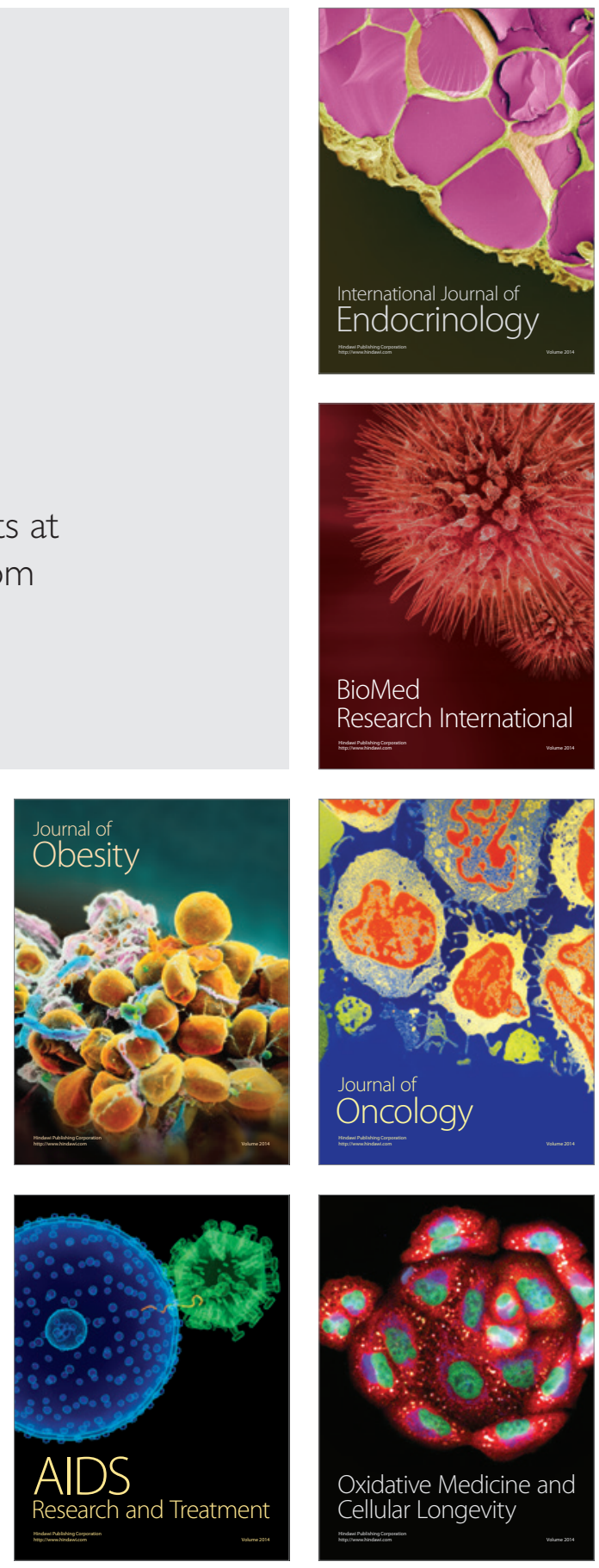